\title{
Age-Related Mitochondrial Changes after Traumatic Brain Injury
}

\author{
Lesley K. Gilmer, ${ }^{1-3}$ Mubeen A. Ansari, ${ }^{1}$ Kelly N. Roberts, ${ }^{1}$ and Stephen W. Scheff ${ }^{1-3}$
}

\begin{abstract}
Mitochondrial dysfunction is known to occur following traumatic brain injury (TBI) and has been well characterized. This study assessed possible age-related changes in the cortical mitochondrial bioenergetics following TBI. Three hours following a moderate TBI, tissue from the ipsilateral hemisphere (site of impact and penumbra) and the corresponding contralateral region were harvested from young (3- to 5-month-old) and aged (22- to 24month-old) Fischer 344 rats. Synaptic and extrasynaptic mitochondria were isolated using a Ficoll gradient, and several bioenergetic parameters were examined using a Clark-type electrode. Injury-related respiration deficits were observed in both young and aged rats. Synaptic mitochondria showed an age-related decline in the rate of ATP production, and a decline in respiratory control ratios (RCR), which were not apparent in the extrasynaptic fraction. Following respiration analysis, mitochondrial samples were probed for oxidative damage (3-nitrotyrosine [3-NT], 4-hydroxynonenal [4-HNE], and protein carbonyls [PC]). All markers of oxidative damage were elevated with injury and age in the synaptic fraction, but only with injury in the extrasynaptic fraction. Synaptic mitochondria displayed the highest levels of oxidative damage and may contribute to the synaptic bioenergetic deficits seen following injury. Data indicate that cortical synaptic mitochondria appear to have an increased susceptibility to perturbation with age, suggesting that the increased mitochondrial dysfunction observed following injury may impede recovery in aged animals.
\end{abstract}

Key words: aging; cortical contusion injury; oxidative damage; respiration; synaptic and extrasynaptic mitochondria; traumatic brain injury

\section{Introduction}

$\mathbf{T}$ RAUMATIC BRAIN INJURY (TBI) is a serious public health care concern affecting over 55 million people worldwide (Langlois et al., 2006), and 1.4 million each year in the United States alone (Thurman et al., 1999). Elderly individuals, 65 years of age or older, account for a disproportionate number of TBI cases and have a staggering incidence of morbidity and mortality (Flaada et al., 2007). Hospitalization and deaths from TBI are higher among older individuals than any other age group (Coronado et al., 2005). TBI among older adults is an under-recognized health problem, even though mortality rates are comparable to those of Alzheimer's disease, diabetes, and influenza (Dellinger and Stevens, 2006), prompting geriatric TBI research.

Many reports confirm that age negatively affects outcome following trauma, both clinically (Mosenthal et al., 2002; Richmond et al., 2002; Vollmer, 1991), and experimentally in ischemia (Yager et al., 2006), intracerebral hemorrhage injury
(Gong et al., 2005), closed head injury (Maughan et al., 2000), and neurotrauma (Cotman and Scheff, 1979; Gong et al., 2005; Hamm et al., 1991; Kovacs, 2005; Maughan et al., 2000; Topp et al., 1989; Zhu et al., 2003). Hukkelhoven and associates (Hukkelhoven et al., 2003) reported that older age is continuously associated with a more negative outcome following TBI, with the probability for poor outcome increasing by $40-$ $50 \%$ for every decade following 39 years of age. Data suggest that as individuals age, they become more susceptible to cellular perturbation or have lowered thresholds for dysregulation, which increase the likelihood of an exacerbated response given the same level of insult.

There have been several studies that have revealed possible explanations as to why older individuals fare worse following TBI. Older patients usually have a higher occurrence of subdural hematomas (Mosenthal et al., 2002; Vollmer et al., 1991) and pre-existing comorbid medical conditions (Richmond et al., 2002), and are often taking multiple medications that can exacerbate injury and compromise recovery. Several other

\footnotetext{
${ }^{1}$ Sanders Brown Center on Aging, ${ }^{2}$ Department of Anatomy and Neurobiology, and ${ }^{3}$ Spinal Cord and Brain Injury Research Center, University of Kentucky, Lexington, Kentucky.
} 
age-related systemic changes that may affect outcome following TBI have been reviewed (Thompson and Bourbonniere, 2006).

One reasonable cellular explanation for some age-related differences may be related to alterations in mitochondrial respiration in the cortex, and from elevated levels of oxidative damage. Several theories of aging implicating mitochondria involve reactive oxygen species (ROS), which are produced during normal cellular respiration, as a possible driving force underlying the aging process. ROS can cause oxidative damage to several components of mitochondria, including the electron transport chain (ETC) (Dencher et al., 2007; Lesnefsky and Hoppel 2006; Van Remmen and Richardson 2001), lipid membranes (Hoch, 1992), and DNA (Richter, 1995). Oxidative damage to mitochondrial components has been shown to accumulate in an age-dependent manner in certain mitochondrial fractions (Gilmer et al., 2010), and can alter several mitochondrial and cellular functions (Stowe, 2009). This accumulation of oxidative damage in mitochondria is associated with an increase in ROS production in several regions of the brain (Driver et al., 2000). Age-related increased oxidative stress alters endogenous antioxidant defenses and leaves the brain more susceptible to oxidative damage (Joseph et al., 1996).

Research has implicated a decline in mitochondrial respiration as a mechanistic explanation for the observed functional decline and cellular senescence exhibited with normal aging in many tissue types (Lesnefsky and Hoppel, 2006). The extent of mitochondrial decline that occurs with normal aging is much less pronounced than that seen in pathological conditions (Finsterer, 2008). There is an ongoing debate concerning the magnitude of decline in mitochondrial respiration that occurs during normal aging (Gilmer et al., 2010). Some groups report extensive age-related declines in mitochondrial respiration (Chiu and Richardson, 1980; Cocco et al., 2005; Navarro et al., 2005, 2008; Petrosillo et al., 2008a,b). Our laboratory and others (Bustamante et al., 2008; Deshmukh et al., 1980; Gilmer et al., 2010; Leslie et al., 1985; Meng et al., 2007) previously reported no significant age-related changes in cortical mitochondrial bioenergetics in naïve rats, while significant elevations in oxidative damage were apparent (Gilmer et al., 2010). It is possible that under naïve conditions the subtle age-related declines in mitochondrial respiration are not sufficient to impair resting energy demands, but perhaps when the system is challenged deficits become more apparent.

Mitochondria display a significant amount of dysfunction early after TBI (Gilmer et al., 2009; Lifshitz et al., 2004; Sullivan et al., 1998, 2002; Xiong et al., 1997). This dysfunction contributes to the progression of several other secondary cascades that ensue for many days after the insult (Siesjo and Siesjo, 1996), particularly since they are the major source of damaging ROS (Hall et al., 2004). The theory suggested by Signorini and associates (1999), that the repair mechanisms of the brain decline with age, may implicate mitochondria as an important variable. Higher levels of oxidative damage to machinery inside mitochondria of aged subjects, particularly in the synaptic fraction (Gilmer et al., 2010), may impede their ability to produce ATP, resulting in greater cellular dysfunction. Increased mitochondrial dysfunction may be a mechanistic explanation as to why aged individuals require longer recovery times and experience increased morbidity and mortality fol- lowing many different neurological insults. This study probed possible age-related declines in mitochondrial respiration, and determined levels of oxidative damage that accumulate following TBI.

\section{Methods}

Male Fischer 344 (F344) rats $(n=40$; National Institute of Aging, Harlan Laboratories, Indianapolis, IN) maintained on a standard diet were used in this study. The animals were housed in group cages (two per cage) with a 12-h light/dark cycle with free access to food and water. All experimental protocols involving animals were approved by the University of Kentucky Animal Use and Care Committee. The rats were assigned to groups based on their age: young: 3-5 months $(n=20)$, and aged: $22-24$ months $(n=20)$. Different age groups were randomly selected each day to ensure consistency of surgeries and mitochondrial isolation procedures.

\section{Surgical procedure}

Cortical contusion injury (CCI) was carried out under isoflurane anesthesia ( $2 \%$ ) as previously described (Gilmer et al., 2009). All injuries were produced using a pneumatic CCI device (TBI 0310; Precision Systems and Instrumentation, Fairfax Station, VA) with a hard stop Bimba cylinder (Bimba Manufacturing, Monee, IL). A moderate injury was given to both young and aged rats $(1.7 \mathrm{~mm}$ cortical displacement depth, $5 \mathrm{~mm}$ impactor tip diameter [beveled], $400 \mathrm{msec}$ dwell time, and $3.5 \mathrm{~m} / \mathrm{sec}$ velocity). A rectal probe and heating blanket were used to maintain body temperature at $37 \pm 0.1^{\circ} \mathrm{C}$ and allowed the animals to survive for $3 \mathrm{~h}$.

\section{Synaptic and extrasynaptic mitochondria isolation}

The animals were briefly gassed with $\mathrm{CO}_{2}$ until flaccid, decapitated, and the brains were rapidly removed. All subsequent procedures were performed on ice, including the entire mitochondrial isolation protocol. A $10-\mathrm{mm}$ punch of the ipsilateral cortex containing injury and penumbra, and corresponding contralateral tissue were collected. To isolate enough mitochondria for respiration analysis and measurement of oxidative damage, tissue was pooled from two animals subjected to identical injury parameters. Mitochondria were isolated separately from the injured (ipsilateral) and uninjured (contralateral) cortex. Respiratory data were collected from either two animals $(n=1)$ or four animals $(n=2)$ on any given day, which always included contralateral mitochondrial samples as a control (synaptic and extrasynaptic) to ensure consistency and quality of isolation procedures. The contralateral sample was used as a baseline to compare respiration changes in the ipsilateral cortex. Previous work has demonstrated that respiration is unaltered in the contralateral hemisphere at $3 \mathrm{~h}$ post-injury, and is equivalent to respiration seen in sham or naïve animals (Gilmer et al., 2009).

Cortical tissue used for respiration analysis was placed in an all-glass dounce homogenizer with $4 \mathrm{~mL}$ of isolation buffer with $1 \mathrm{mM}$ EGTA ( $215 \mathrm{mM}$ mannitol, $75 \mathrm{mM}$ sucrose, $0.1 \%$ defatted bovine serum albumin (BSA), $20 \mathrm{mM}$ HEPES, and $1 \mathrm{mM}$ EGTA [pH 7.2]). The samples were homogenized and mitochondria were extracted by differential centrifugation. 
The homogenate was spun twice at $1300 \mathrm{~g}$ for $3 \mathrm{~min}$ in an Eppendorf microcentrifuge at $4{ }^{\circ} \mathrm{C}$, and the supernatant was transferred to new tubes. The resulting supernatant was topped off with isolation buffer with EGTA and spun at $13,000 \mathrm{~g}$ for $10 \mathrm{~min}$. The supernatant was discarded; the pellet was resuspended with isolation buffer with EGTA to a total volume of $500 \mu \mathrm{L}$, and the samples were place on a discontinuous Ficoll gradient (F5415 Ficoll solution type 400, 20\% in $\mathrm{H}_{2} \mathrm{O}$; Sigma-Aldrich, St. Louis, MO), composed of two layers ( $2 \mathrm{~mL}$ of $7.5 \%$ on top of $2 \mathrm{~mL}$ of $10 \%$ Ficoll cut with isolation buffer with EGTA). The final volume of the gradient was $\sim 4.5 \mathrm{~mL}$ in the 7-mL Beckman tubes (344057; Beckman Coulter, Fullerton, CA). The samples were placed in a Beckman SW $55 \mathrm{Ti}$ swinging bucket rotor and centrifuged at $32,000 \mathrm{rpm}$ for $30 \mathrm{~min}$ at $4^{\circ} \mathrm{C}$. The gradient produced two separate mitochondrial fractions: the extrasynaptic mitochondrial pellet (bottom), and synaptic mitochondria trapped in synaptosomes located at the interface of the two Ficoll layers. Synaptosomes were collected in 2.5-mL tubes, resuspended, and washed in isolation buffer with EGTA by centrifugation at $13,000 \mathrm{~g}$ for $10 \mathrm{~min}$ to remove Ficoll from the sample. The extrasynaptic mitochondrial pellet was collected in 500- $\mu \mathrm{L}$ tubes and also resuspended and washed using isolation buffer with EGTA. Both fractions were washed with fresh buffer a second time to ensure that the Ficoll was eliminated. Synaptosomes were resuspended in isolation buffer with EGTA, and mitochondria were released using a nitrogen cell disruption bomb made by Parr Instrument Company (Moline, IL) at 1200 psi for $10 \mathrm{~min}$. Disrupted synaptosomes were placed on a new Ficoll gradient and returned to the ultracentrifuge and spun at $32,000 \mathrm{rpm}$ for $30 \mathrm{~min}$ at $4{ }^{\circ} \mathrm{C}$. After removal of the Ficoll gradient, the synaptic mitochondria pellet was resuspended in isolation buffer with EGTA and centrifuged at $13,000 \mathrm{~g}$ for $10 \mathrm{~min}$. Both mitochondrial fractions (synaptic and extrasynaptic) were resuspended with isolation buffer without EGTA and centrifuged at 10,000 $g$ for $10 \mathrm{~min}$ to wash out the calcium chelator (EGTA). The final mitochondrial pellets were resuspended in isolation buffer without EGTA to yield a concentration of $10 \mathrm{mg} / \mathrm{mL}$ or higher. Protein concentration was determined using the bicinchoninic acid protein assay kit from Pierce Protein Research Products (Rockford, IL) by measuring absorbance at $560 \mathrm{~nm}$ with a microplate reader (Molecular Devices Corp., Sunnyvale, CA).

\section{Mitochondrial respiration measurements}

Mitochondrial functionality was assessed using an Oxytherm Clark-type oxygen electrode (OXYT1/ED; Hansatech Instruments, Norfolk, U.K.). Mitochondria ( $\sim 60 \mu \mathrm{g}$ isolated from the contralateral cortex and $\sim 68 \mu \mathrm{g}$ from ipsilateral tissue) were placed in the sealed Oxytherm chamber containing respiration buffer $(125 \mathrm{mM} \mathrm{KCl}, 0.1 \%$ BSA, $20 \mathrm{mM}$ HEPES, $2 \mathrm{mM} \mathrm{MgCl}_{2}$, and $2.5 \mathrm{mM} \mathrm{KH}_{2} \mathrm{PO}_{4}$ [pH 7.2]), and continuously stirred at $37^{\circ} \mathrm{C}$. To ensure that all preparations contained equal amounts of respiring mitochondria, a standardized state II respiration rate was used as baseline ( 10-15 $\mathrm{nmol}$ oxygen $/ \mathrm{min}$ ).

For each state of respiration, the rate of oxygen consumption was defined as the slope of the response of the mitochondria to consecutive administrations of respiration substrates, as previously described (Gilmer et al., 2009) with minor modifications. The amount of oligomycin administered was $0.4 \mu \mathrm{L}$, and two additions of adenosine diphosphate (ADP) were administered to ensure accurate state III respiration measurements. The modified protocol of the substrates injected into the Oxytherm chamber sequentially were as follows: $2.5 \mu \mathrm{L}$ of pyruvate/malate (P/M; $2.5 \mathrm{mM}), 1.25 \mu \mathrm{L}$ of ADP $(150 \mu \mathrm{M})$ added twice at 1-min intervals, $0.4 \mu \mathrm{L}$ oligomycin $(1 \mu \mathrm{M}), 1 \mu \mathrm{L}$ carbonylcyanide-4-(trifluoromethoxy)phenylhydrazone (FCCP; $1 \mu \mathrm{M}) ; 0.2 \mu \mathrm{L}$ rotenone $(1 \mu \mathrm{M})$ was added to shut down complex 1 activity, resulting in cessation of oxygen utilization; and $2.5 \mu \mathrm{L}$ succinate $(10 \mathrm{mM})$ was added.

The overall oxygen utilization rate was determined by measuring the amount of oxygen consumed throughout all states of respiration divided by the time elapsed and amount of mitochondrial protein present in the assay. This measurement serves as an index of their overall respiration capacities. States II-V are mitochondrial respiration parameters driven by complex $1(\mathrm{P} / \mathrm{M}, \mathrm{ADP}$, oligomycin, and FCCP), while state $\mathrm{V}$-succinate represents maximum complex 2-driven respiration. Maximum respiration capabilities were quantified by supplying the ETC with all the necessary substrates, then bypassing the restricting ATP synthase via FCCP. Addition of FCCP, a pure uncoupler that acts as a protonophore, allowed protons in the inner membrane space to freely pass into the matrix. The ETC tries to maintain the proton gradient, which results in a high level of oxygen utilization during state $\mathrm{V}$ respiration. Following addition of FCCP, rotenone was added to specifically inhibit complex 1 to allow for measurement of maximum complex 2-driven respiration independently. Inhibiting complex 1 respiration increases the signal-to-noise ratio during complex 2 state $\mathrm{V}$ respiration. The respiratory control ratio (RCR) was determined by dividing the rate of oxygen utilization for state III (ADP) by state IV (oligomycin). RCR values indicate how coupled the ETC is to ATP production.

\section{Markers of oxidative damage quantification}

Estimation of protein carbonylation. Following respiration analysis, unused synaptic and extrasynaptic mitochondria samples were probed for levels of oxidative damage. Levels of protein carbonyls (PC) were assessed as described previously (Ansari et al., 2008a). All samples were normalized to $4 \mathrm{mg} / \mathrm{mL}$, then combined with sodium dodecyl sulfate (SDS) and dilute 2,4-dinitrophenyl hydrazine. Samples were incubated at room temperature for $20 \mathrm{~min}$ then neutralized with $2 \mathrm{M}$ Tris in $30 \%$ glycerol. The resulting samples were loaded on a nitrocellulose membrane and run on a slot-blot apparatus. The membranes were blocked in 3\% BSA and PBS/Tween for $1 \mathrm{~h}$, and incubated with a 1:100 dilution of anti-DNP polyclonal antibody in PBS/Tween for $1 \mathrm{~h}$. The membranes were washed in PBS/Tween and incubated for $1 \mathrm{~h}$ with an anti-rabbit IgG alkaline phosphatase secondary antibody diluted in PBS/Tween at a 1:8000 ratio. Each membrane was washed in PBS/Tween and developed in Sigma Fast tablets. The blots were dried, scanned with Adobe Photoshop, and quantified with Scion Image. There was no nonspecific binding of antibody to the membrane observed. The intensity of PC staining was quantified by optical density and data are reported as percentage of young values from the uninjured hemisphere. 
Estimation of 3-nitrotyrosine (an index of protein nitration), and 4-hydroxynonenal (an index of lipid peroxidation). Ficoll purified synaptic and extrasynaptic mitochondria samples were normalized with $12 \%$ SDS and modified Laemmli buffer containing $0.125 \mathrm{M}$ Tris base [pH 6.8], 4\% (v/v) SDS, and $20 \%(\mathrm{v} / \mathrm{v})$ glycerol, and were incubated for $20 \mathrm{~min}$ at room temperature. Samples (250 ng) were loaded into wells on a nitrocellulose membrane in a slot-blot apparatus under vacuum. The membranes were blocked with $3 \%$ BSA in PBS/Tween for $1 \mathrm{~h}$, and then incubated with either a 1:2000 dilution of anti-3nitrotyrosine (3-NT) polyclonal antibody, or a 1:5000 dilution of anti-HNE polyclonal antibody in PBS/Tween for $1.5 \mathrm{~h}$. Each membrane was washed in PBS/Tween for 5 min three times after incubation. The membranes were incubated for $1 \mathrm{~h}$ after washing with an anti-rabbit IgG alkaline phosphatase secondary antibody diluted in PBS/Tween at a 1:8000 ratio. The membranes were washed three times in PBS/Tween for $5 \mathrm{~min}$ and developed with Sigma Fast tablets. The blots were dried, scanned with Adobe Photoshop, and quantified with Scion Image as above. Nonspecific binding of antibody to the membrane was not observed. The intensities of 4-HNE and 3-NT staining were quantified by optical density, and data are reported as percent of young values from the uninjured hemisphere.

\section{Statistical analysis}

Data from mitochondrial fractions (synaptic and extrasynaptic) were expected to be different based on previous literature demonstrating structural and functional differences in these fractions (Brown et al., 2006; Davey and Clark, 1996; Davey et al., 1997, 1998; Erecinska et al., 1996; Naga et al., 2007). All data are reported as group means \pm SD. A two-way repeated-measures analysis of variance (ANOVA) (age $\times$ hemisphere) was used to compare the amounts of mitochondrial protein used for respiration analysis of each fraction separately.

All respiration parameters for each sample were assessed multiple times, and the average values were used in the final analysis. There was not enough sample to verify respiration findings on three occasions, so samples were excluded from analysis (1 young synaptic, 1 aged synaptic, and 1 aged extrasynaptic). Actual values were reported for both overall oxygen utilization rate and RCR values, since they are calculated measurements that are obtained from two or more states of respiration. These two measurements were analyzed with a two-way repeated-measures ANOVA (age $\times$ mitochondrial fraction) for both the ipsilateral and contralateral hemispheres. A Student-Newman-Keuls post-hoc analysis was used with alpha set at 0.05 . Previous work by our laboratory has shown that mitochondrial respiration does not decline with normal aging in F344 rats (Gilmer et al., 2010), therefore respiration data collected from the contralateral hemisphere of young and aged rats were used as internal controls, respectively.

All other states of respiration (II, III, IV, V-FCCP, and $\mathrm{V}$-succinate) were measured as nanomoles of oxygen utilized/ $\mathrm{min} / \mathrm{mg}$ of mitochondrial protein, and then normalized as a percentage of the contralateral rate for each respiration state. Reporting data as the percentage of the contralateral respiration rate minimizes biological variance, and serves as an appropriate internal control for normal respiration capabilities (Gilmer et al., 2009). Using percentage of the contralateral value also ensures that possible age-related changes are not masked by a possible injury effect. Measurements of bioenergetics were analyzed with a two-way repeated-measures ANOVA (age $\times$ mitochondrial fraction). A Student-Newman-Keuls posthoc analysis was used with alpha set at 0.05 .

Markers of oxidative damage (4-HNE, 3-NT, and PC) were measured in both synaptic and extrasynaptic mitochondrial fractions and analyzed separately. The levels of oxidative damage were expressed as the percentage of that observed in the uninjured hemisphere of young animals. A two-way repeated-measures ANOVA (age $\times$ hemisphere) was used to determine the extent of oxidative damage following injury. A Student Newman-Keuls post-hoc test was used with alpha set at 0.05 . When appropriate, possible differences between synaptic and extrasynaptic mitochondrial fractions were evaluated with a paired Student's $t$-test and a Bonferroni correction factor for multiple comparisons.

\section{Results}

\section{Mitochondrial amounts used for respiration analysis}

The average amounts of extrasynaptic mitochondrial protein used to assess respiration capabilities from young animals were $68.5 \pm 15.9 \mu \mathrm{g}$ (contralateral) and $92.2 \pm 21.6 \mu \mathrm{g}$ (ipsilateral), and for aged rats were $67.8 \pm 8.9 \mu \mathrm{g}$ (contralateral) and $94.0 \pm 12.0 \mu \mathrm{g}$ (ipsilateral). A two-way repeatedmeasures ANOVA (age $\times$ hemisphere) revealed a significant main effect for hemisphere $[F(1,17)=24.982, p<0.0001]$, but not for age $[F(1,17)=0.012, p<0.1]$. Post-hoc testing (paired $t$-test) revealed that significantly more mitochondria $(p<0.001)$ were used for respiration in the ipsilateral (injured) hemisphere than in the uninjured hemisphere. The average amounts of synaptic mitochondrial protein used to assess respiration capabilities from young animals were $64.9 \pm 11.21 \mu \mathrm{g}$ (contralateral) and $81.8 \pm 15.7 \mu \mathrm{g}$ (ipsilateral), and for aged rats were $54.1 \pm 7.6 \mu \mathrm{g}$ (contralateral) and $62.6 \pm 13.7 \mu \mathrm{g}$ (ipsilateral). A two-way repeated-measures ANOVA (age $\times$ hemisphere) revealed a significant main effect for hemisphere $[F(1,17)=14.188, p<0.002]$, and also for age $[\mathrm{F}(1,17)=10.480, p>0.01]$. However, there was no significant interaction between hemisphere and age. Similarly to the extrasynaptic findings, post-hoc testing (paired $t$-test) revealed that significantly greater amounts of mitochondria $(p<0.001)$ were used in the analysis of the injured hemisphere.

Figure 1 shows distinct respiration profiles in both mitochondrial fractions (synaptic and extrasynaptic) for both young and aged animals expressed as nanomoles of oxygen utilized $/ \mathrm{min} / \mathrm{mg}$ protein. Both age groups displayed similar profiles following injury. It is important to note that the baseline levels for the contralateral hemisphere for the synaptic fractions are significantly lower than the extrasynaptic fraction, indicating a significant difference in respiration between these two mitochondrial fractions, as previously described (Gilmer et al., 2010).

Overall oxygen utilization rate. Significantly lower overall oxygen utilization rates were apparent in the ipsilateral hemisphere compared to the contralateral hemisphere [F $(1,17)=114.51, p<0.0001$; Fig. 2a). Following TBI there are significant declines in overall oxygen utilization rates in both mitochondrial fractions. A repeated-measures ANOVA was applied to the ipsilateral hemisphere (age $\times$ mitochondrial fraction). While there was no effect of age $[F(1,17)=1.048, p>0.1]$, there was a significant main effect 


\section{A Young rats \\ Early respiration changes following TBI}
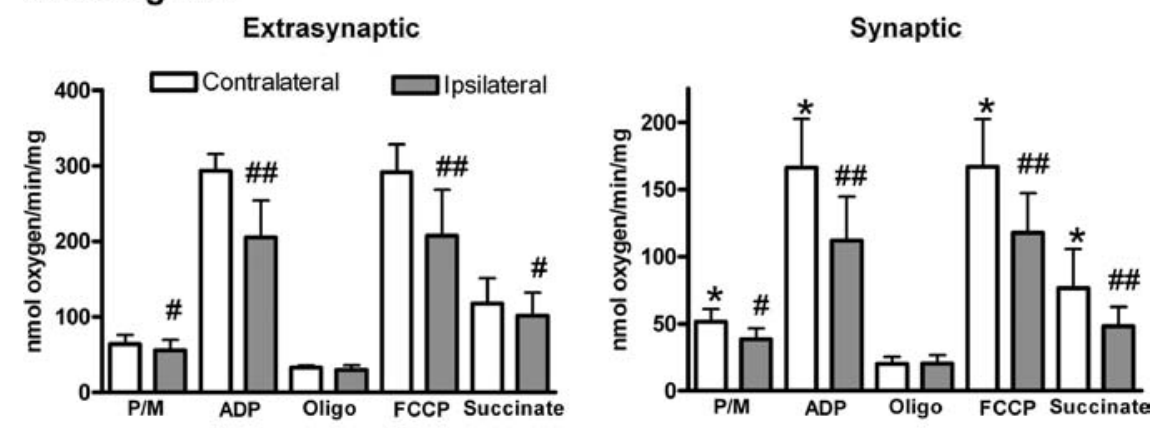

\section{B Aged rats}
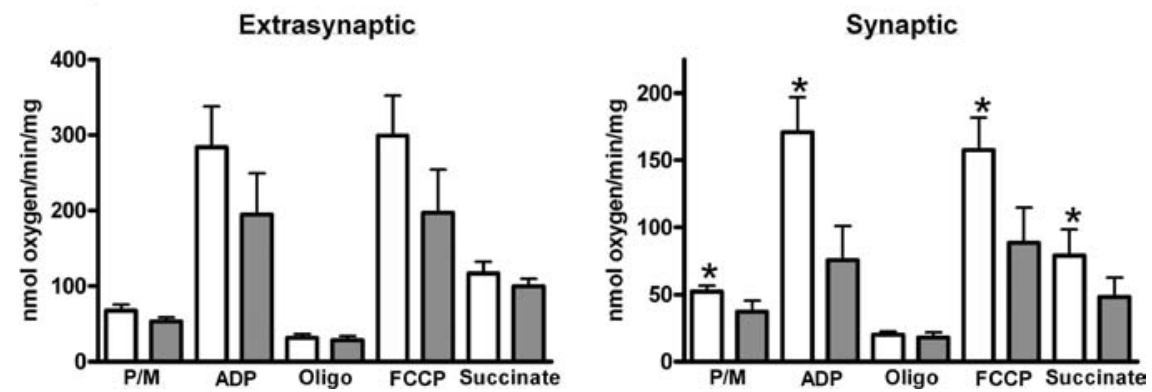

FIG. 1. Quantification of all states of respiration for both (A) young and (B) aged rats for both the extrasynaptic and synaptic mitochondrial fractions. No significant age-related changes were apparent in any respiration parameter in extrasynaptic mitochondria, but all respiration states were significantly higher than the synaptic fraction. The range of the respiration responses in the extrasynaptic fraction are significantly higher than those in the synaptic fraction, indicating a baseline shift in respiration between these two fractions [bars represent group means \pm standard deviation; ${ }^{*} p<0.05$ compared to extrasynaptic values; ${ }^{\#} p<0.05 ;{ }^{\# \#} p<0.01$ compared to contralateral values; $\mathrm{P} / \mathrm{M}$, pyruvate/malate; ADP, adenosine diphosphate; Oligo, oligomycin; FCCP, carbonylcyanide-4-(trifluoromethoxy)-phenylhydrazone].

for fraction $[F(1,17)=188.56, p<0.0001]$. Post-hoc analysis revealed that extrasynaptic mitochondria display significantly higher overall oxygen utilization rates than the synaptic fraction, regardless of age. A repeated-measures ANOVA was also applied to the contralateral hemisphere data and revealed a significant main effect for mitochondrial fraction $[\mathrm{F}(1,17)=236.23, p<0.0001]$, but no significant main effect for age $[F(1,17)=0.486, p>0.1]$. The post-hoc analysis again revealed that extrasynaptic mitochondria displayed significantly higher overall oxygen utilization rates.

Pyruvate/malate respiration (state II via complex I). State II respiration ( $\sim 15$ nmoles oxygen $/ \mathrm{min})$ was used as a baseline that gave all groups an equal ability to produce similar respiration profiles, regardless of the amount of mitochondrial protein added. The percentages of contralateral state II respiration rates were compared with a repeated-measures ANOVA (age $\times$ mitochondrial fraction). The analysis failed to reveal a significant main effect for age [F $(1,17)=0.758, p>0.1]$, or for mitochondrial fraction $[\mathrm{F}(1,17)=2.759, p>0.1]$.

ADP respiration (state III). Figure $2 c$ shows changes in state III respiration (as a percentage of the contralateral hemisphere) following injury in both fractions of mitochondria. A repeated-measures ANOVA (age $\times$ mitochondrial fraction) revealed a significant main effect for fraction $[F(1,17)=11.26, p<0.005]$, but no significant main effect for age $[\mathrm{F}(1,17)=4.42, p>0.05]$. However, there was a significant age $\times$ mitochondrial fraction interaction $[F(1,17)=7.28$, $p<0.05]$. Post-hoc analysis revealed that ADP rates in the synaptic fraction of aged subjects was significantly lower than rates observed in the younger cohorts. In addition, synaptic rates were lower than extrasynaptic rates, regardless of age.

Oligomycin respiration (state IV). Changes in state IV respiration rates (as a percentage of the contralateral hemisphere) following TBI are depicted in Figure $2 \mathrm{~d}$. A repeatedmeasures ANOVA (age $\times$ mitochondrial fraction) revealed no main effect for age $[F(1,17)=0.317, p>0.1]$, or for mitochondrial fraction $[\mathrm{F}(1,17)=0.532, p>0.1]$.

RCR (state III/state IV respiration). The RCR is an index of how coupled the ETC is to ATP production. Figure $2 b$ shows alterations in RCR values following injury in both fractions of mitochondria. A repeated-measures ANOVA (age $\times$ mitochondrial fraction) in the ipsilateral hemisphere revealed a significant main effect for age $[F(1,17)=10.051$, $p<0.01]$, and a significant main effect for mitochondrial fraction $[F(1,17)=156.38, p<0.0001]$. There was also a significant interaction between age and mitochondrial fraction [F $(1,17)=17.101, p<0.001]$. Post-hoc testing revealed that extrasynaptic mitochondria display significantly higher RCR values than those observed in the synaptic fraction. Synaptic mitochondria in the aged cohort displayed significantly lower RCR values than the younger subjects. A separate analysis 
A Overall oxygen utilization rate

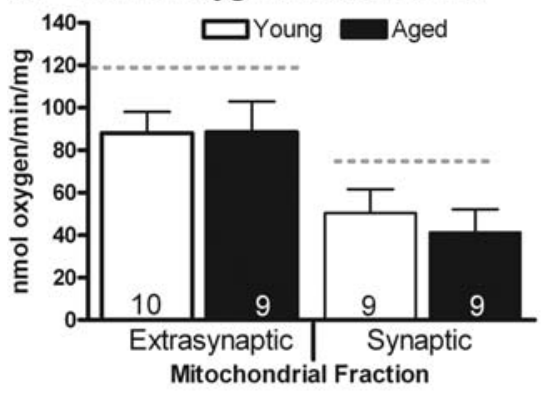

C ADP respiration

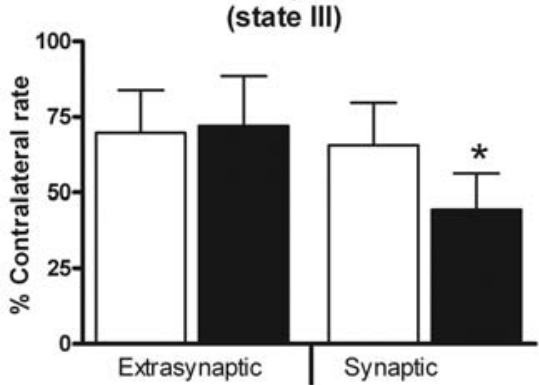

E

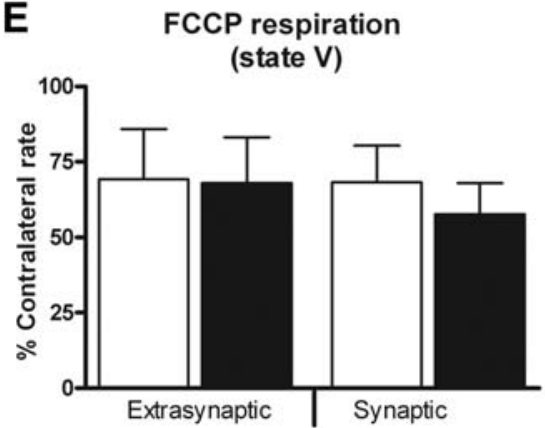

B Respiratory control ratio
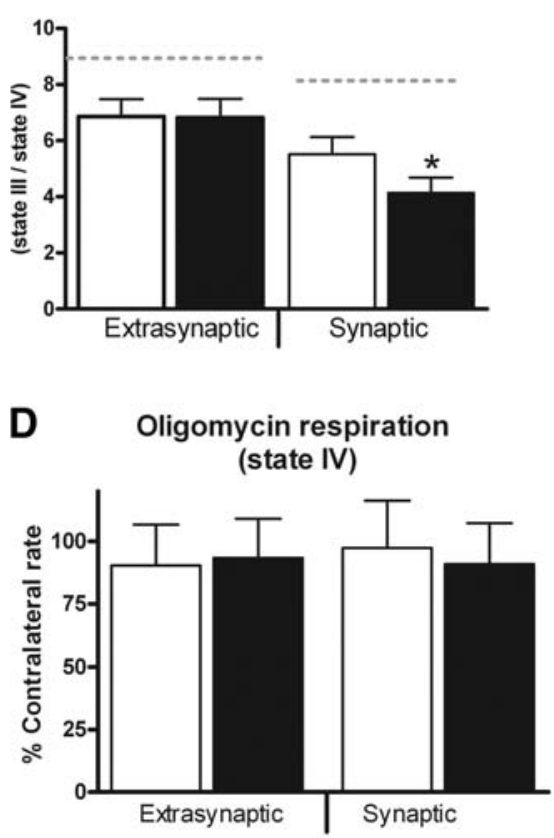

$\mathbf{F}$

Succinate respiration (state V)

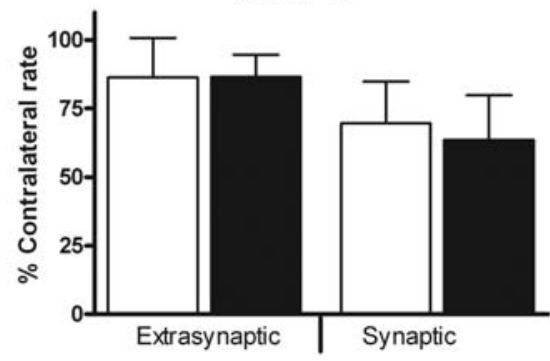

FIG. 2. Mitochondrial bioenergetic assessment in cortical samples ipsilateral to the injury. (A) Overall oxygen utilization rate. The dashed line represents the overall oxygen utilization rates from the contralateral hemisphere. No significant age-related changes were apparent in this respiration parameter in either mitochondrial fraction. A significant difference between these two mitochondrial fractions did exist. The subtle decline seen in the synaptic fraction of aged animals may be indicative of changes in only certain states of respiration. (B) Respiratory control ratio (RCR). RCR values are used as a general index of how coupled respiration is to ATP production. The dashed line indicates the RCR value in the contralateral hemisphere. Mitochondria are severely dysfunctional and respiration becomes uncoupled from ATP production when RCR values drop below 5 . RCR values declined significantly as a result of the injury in both mitochondrial fractions, but only synaptic mitochondria became severely dysfunctional. These data suggest that extrasynaptic mitochondria are able retain their ability to efficiently produce ATP for at least $3 \mathrm{~h}$ post-injury, while synaptic mitochondria have already become dysfunctional. Percentages of contralateral respiration rates for synaptic and extrasynaptic fractions are shown in: (C) state III-ADP, (D) state IV-oligomycin, (E) state V-FCCP, and (F) state V-succinate (bars represent group means \pm standard deviation; ${ }^{*} p<0.05$ compared to young values).

revealed that mitochondria taken from the injured hemisphere displayed significantly lower RCR values than the contralateral side $[\mathrm{F}(1,17)=466.79, p<0.0001]$.

FCCP respiration (state V). Figure $2 \mathrm{e}$ depicts the alterations (as a percentage of the contralateral hemisphere) to state $\mathrm{V}$ respiration following injury. A repeated-measures ANOVA (age $\times$ mitochondrial fraction) failed to reveal a significant main effect for age $[F(1,17)=2.235, p>0.1]$, or for mitochondrial fraction $[\mathrm{F}(1,17)=1.272, p>0.1]$.

Succinate respiration (state $\mathrm{V}$ via complex 2). Changes in complex 2 state $\mathrm{V}$ respiration rates (as a percentage of the con- tralateral hemisphere) following TBI are depicted in Figure $2 \mathrm{f}$. A repeated-measures ANOVA (age $\times$ mitochondrial fraction) revealed a significant main effect for mitochondrial fraction $[F(1,17)=24.872, p<0.0001]$, but not for age $[F(1,17)=0.073$, $p>0.1]$. Post-hoc analysis revealed that extrasynaptic mitochondria displayed significantly higher state V-succinate respiration than the synaptic fraction, regardless of age.

\section{Markers of oxidative damage}

Protein carbonyls. An analysis of the extrasynaptic fraction using a two-way repeated-measures ANOVA (hemisphere $\times$ age) revealed a significant elevation in the levels of 
oxidized proteins in the injured hemisphere $[\mathrm{F}(1,18)=59.111$, $p<0.0001]$, but failed to demonstrate a main effect for age $[F(1,18)=1.669, p>0.1$; Fig. 3a]. A similar analysis of the synaptic fraction demonstrated a significant hemisphere elevation in PC $[F(1,18)=29.842, p<0.001]$, and also a main effect for age $[\mathrm{F}(1,18)=15.624, p<0.001]$. Post-hoc testing revealed significantly greater levels of PC in the injured hemisphere for both age groups. A paired $t$-test failed to demonstrate a difference in the levels of PC between synaptic and extrasynaptic fractions $\left[t_{19}=1.12, p>0.1\right]$.

4-Hydroxynonenal. An analysis of the 4-HNE levels in the extrasynaptic fraction using a two-way repeated-measures ANOVA (age $\times$ hemisphere) revealed a significant elevation in the injured hemisphere $[\mathrm{F}(1,18)=9.693, p<0.01]$, but failed to demonstrate a main effect for age $[\mathrm{F}(1,18)=3.938, p>0.05$; Fig. 3b). Post-hoc testing showed that the injured hemisphere had significantly higher 4-HNE levels. A similar analysis carried out on the synaptic fraction demonstrated a significant effect for hemisphere $[\mathrm{F}(1,18)=16.516, p<0.001]$, and also a main effect for age [F $(1,18)=10.708, p<0.005]$. Post-hoc testing revealed significantly higher levels in the injured hemisphere, and significantly higher levels in the aged animals. A paired $t$-test demonstrated a significant difference in the levels of 4-HNE between synaptic and extrasynaptic fractions $\left[t_{19}=2.36, p<0.05\right]$, with the synaptic fraction having increased levels.

3-Nitrotyrosine. 3-NT was used as a biomarker of reactive nitrogen species formation (Walker et al., 2001), specifically peroxynitrite-induced protein modification (Kaufmann et al., 2001). An analysis of the 3-NT levels in the extrasynaptic fraction, using a two-way repeated-measures ANOVA (age $\times$ hemisphere), revealed a significant elevation in the injured hemisphere $[F(1,18)=7.571, p<0.01]$, but failed to demonstrate a main effect for age $[\mathrm{F}(1,18)=1.741, p>0.1$; Fig. 3c]. Post-hoc testing revealed significantly higher levels in the injured hemisphere. A similar analysis of the 3-NT levels in the synaptic fraction demonstrated a significant hemisphere elevation in 3-NT [F $(1,18)=6.037, p<0.05]$, and also a main effect for age $[\mathrm{F}(1,18)=9.775, p<0.01]$. Post-hoc testing revealed significantly higher levels in the older animals, and significantly higher levels in the injured hemisphere. Synaptic mitochondria displayed significantly higher levels of 3-NT than the extrasynaptic fraction [paired $t_{19}=2.69, p<0.01$ ].

\section{Discussion}

This is the first study to fully characterize injury-induced age-related changes in mitochondrial bioenergetics in the cortex. Aged F344 rats subjected to a moderate TBI demonstrated a significant decline in ATP production capabilities in the synaptic mitochondrial fraction compared to younger rats subjected to the same injury. This mitochondrial fraction also showed significant declines in RCR values, a general measurement of mitochondrial functionality. The present set of experiments differs from previous TBI studies in that both synaptic and extrasynaptic mitochondria were analyzed separately. Enriched extrasynaptic fractions are primarily composed of mitochondria from neuronal cell bodies, axons, and dendrites, as well as support cells (astrocytes and microglia), while synaptic fractions only contain mitochondria
A

Protein carbonyls

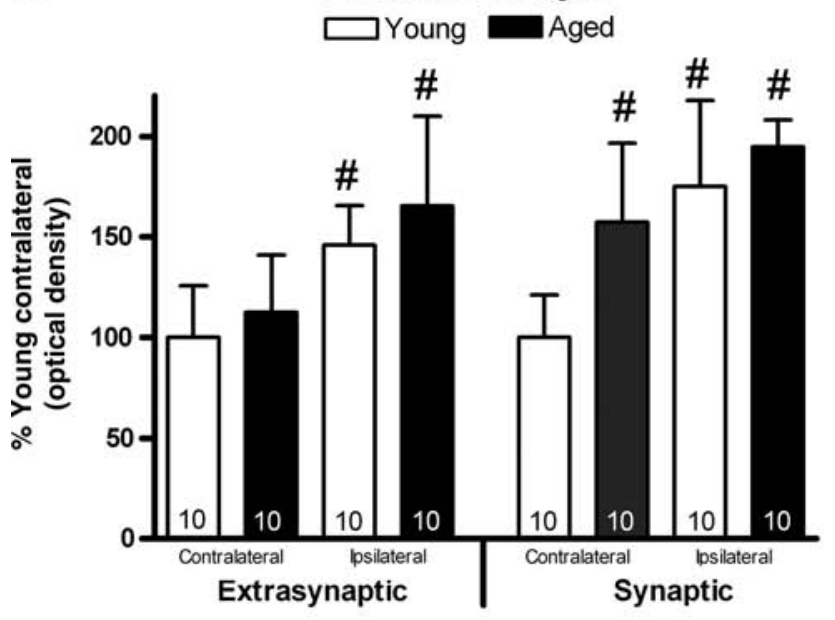

B 4-Hydroxynonenal
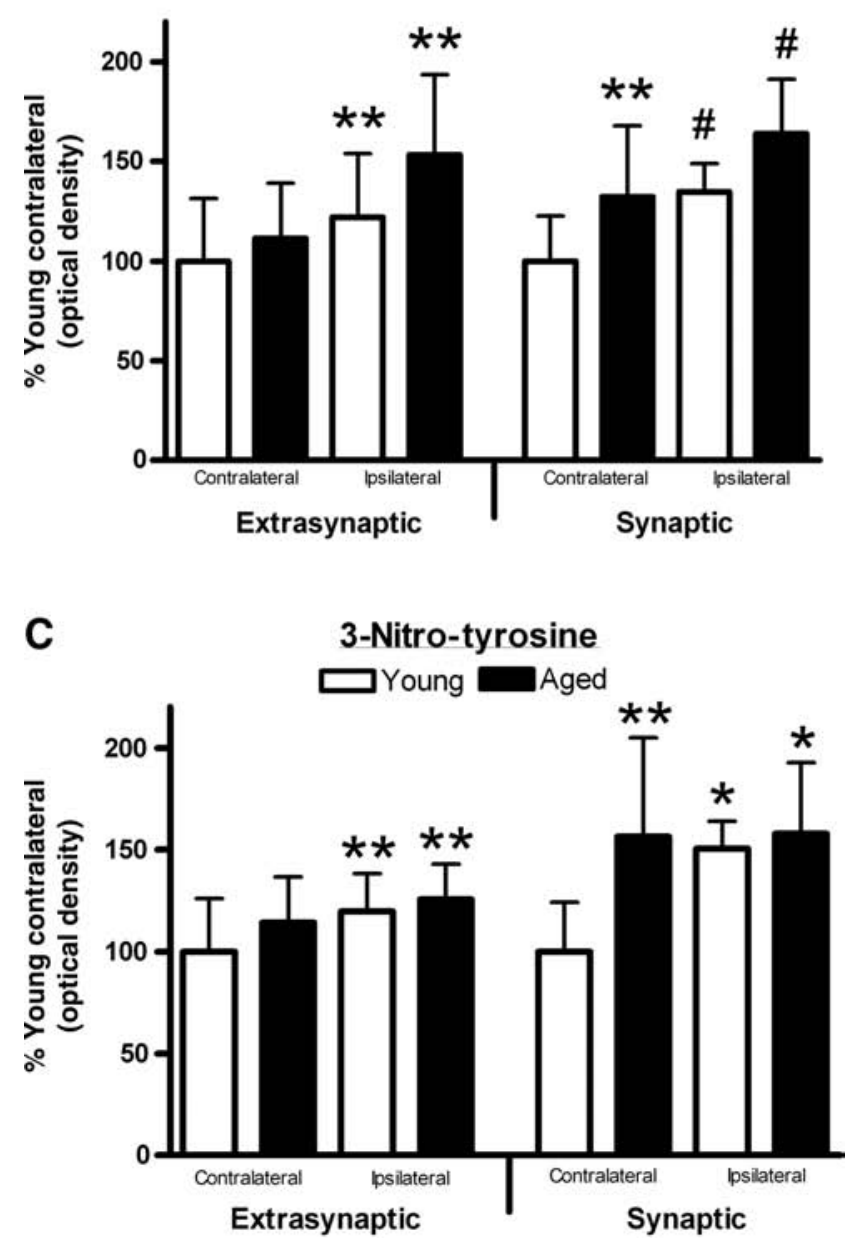

FIG. 3. Changes in measures of oxidative stress following injury. The levels of oxidized proteins, as measured by levels of protein carbonyls (A), 4-hydroxynonenal (4-HNE)-protein adducts (B), and 3-nitrotyrosine (3-NT), a biomarker of reactive nitrogen species formation $(\mathbf{C})$ were determined in purified synaptic and extrasynaptic mitochondrial fractions (bars represent group means \pm standard deviation; ${ }^{*} p<0.05$; ${ }^{* *} p<0.01 ;{ }^{*} p<0.001$ compared to young animals). 
from pre- and post-synaptic terminals. These experiments also revealed an increase in oxidative damage, with the greatest changes occurring in the synaptic fraction. Synaptic mitochondria from aged animals may be burdened with elevated oxidative damage to their mitochondrial machinery prior to injury, resulting in an exacerbation of dysfunction when the system is stressed. Extrasynaptic mitochondria do not display elevated levels of oxidative stress with age (Gilmer et al., 2010), supporting previous findings indicating that this fraction may be better equipped to compensate for oxidative damage.

Clinical data strongly suggest that an individual's age at the time of injury negatively correlates with positive outcome, but positively correlates with increased mortality (Utomo et al., 2009). However, there is considerable disagreement as to the critical age at which these changes begin to have a significant influence (Chesnut et al., 2000; Coronado et al., 2005; Flaada et al., 2007; Hukkelhoven et al., 2003; Jacoby et al., 2006; Mosenthal et al., 2002, 2004; Richmond et al., 2002; Rothweiler et al., 1998; Thompson et al., 2006; Vollmer et al., 1991). Initial injury severity may be a more important variable in determining the extent of recovery than age alone, with older patients showing significantly higher mortality rates compared to younger individuals when the injury was moderate to severe (Mosenthal et al., 2004). It has been suggested that with increased age the central nervous system's ability to cope with increased physiological stress following a severe insult is significantly diminished (Signorini et al., 1999; Victorino et al., 2003).

Similar to the clinical data, age-related deficits are also observed following experimental neurotrauma, with results varying by the type of injury administered, the severity of the insult, the age of animal, and the outcome measurements collected. There is ongoing research in experimental TBI to define the age threshold and injury severity that coincides with findings of higher morbidity and mortality. Cognitive and morphological differences have been previously noted in aged experimental brain-injured rats (Fan et al., 2003; Hamm et al., 1991; Hoane et al., 2004; Shah et al., 2006; Shao et al., 2006), and also if injury occurs in the very early stages of development (Casey et al., 2008; Robertson et al., 2007, 2009; Scafidi et al., 2009).

Deterioration of mitochondrial function has been proposed as a contributor to the aging process (Merry, 2004; Nicholls, 2004; Toescu and Verkhratsky, 2003; Trifunovic and Larsson, 2008), and has been well-characterized in pathological conditions (Atamna and Frey, 2007; Beal, 2005; Fukui and Moraes, 2008; Swerdlow, 2007). Age-related oxidative damage could accumulate in proteins responsible for importing substrates inside mitochondria, affecting enzymes needed to start the tricarboxylic acid cycle, such as pyruvate dehydrogenase (Long et al., 2006), various complexes of the ETC (Navarro and Boveris, 2007; Sastre et al., 2003), or the exchange of ADP for ATP across the inner mitochondrial membrane by the adenine nucleotide translocator (Feng et al., 2008; Gouspillou et al., 2009). Any alterations to these structures required for energy production could hinder normal respiration capacity, or their ability to recover when the system is challenged. Recently, we showed that naïve F344 rats display no age-related deficits in mitochondrial respiration (Gilmer et al., 2010), therefore deficits may not be apparent until the mitochondria are challenged. Other data support these findings in F344 rats, including those that show that mitochondrial membrane potential and susceptibility to permeability transition was stable out to 24 months (LaFrance et al., 2005).

We have previously reported early mitochondrial bioenergetic changes following a moderate cortical contusion (Gilmer et al., 2009). The present results confirmed and refined those findings by demonstrating that different mitochondrial fractions respond differently to the insult. While both synaptic and extrasynaptic fractions demonstrated significant reductions in respiration following the trauma, synaptic mitochondria appear to sustain the greatest dysfunction. This is not surprising since prior studies have reported different respiration capacities in these two fractions in naïve rats (Brown et al., 2006; Davey et al., 1997; Gilmer et al., 2010; Naga et al., 2007). Our analyses clearly demonstrate that when the fractions are collapsed across age groups, the injured extrasynaptic fraction is capable of significantly better respiration.

Numerous factors may have played a role in the greater dysfunction seen in synaptic mitochondria following injury. Greater membrane disruption may have occurred at the synaptic terminal at $3 \mathrm{~h}$ post-injury than in other regions of the neuron, leading to more damaged fragments that are similar in size, thus contaminating the mitochondrial preparation. Synaptic mitochondria may have simply sustained more damage to their ETC protein complexes due to their specialized role in the synaptic terminal. Excitotoxicity, calcium overload, and the ensuing increased oxidant production (Greve and Zink, 2009; Marklund et al., 2006; Werner and Engelhard, 2007) may be more severe in the synapses following TBI due to disrupted axoplasmic flow (Adams et al., 1982; Buki et al., 1999; Povlishock and Christman, 1995; Povlishock et al., 1999).

The overall oxygen utilization rate monitors overt changes in respiration. A significant decline in this respiration parameter was found in both mitochondrial fractions in the injured hemisphere, but failed to show any age-related difference. Both mitochondrial fractions contribute to this measure of bioenergetic integrity. The overall oxygen utilization rate in the extrasynaptic fraction of the injured cortex declined by approximately $25 \%$, with the two age groups showing almost identical declines. The synaptic fraction manifested a $38 \%$ decline in oxygen utilization. Although differences between age groups in this fraction did not reach statistical significance, older subjects demonstrated a $45 \%$ decline, compared to a $32 \%$ decline in the younger cohort. These data again support the idea that synaptic mitochondria are more sensitive to the perturbation.

Following TBI, state III respiration was reduced by approximately $30 \%$ in extrasynaptic mitochondria in both age groups, while synaptic mitochondria displayed even greater declines, with older animals demonstrating a 55\% loss of function. The age-related decline in synaptic state III respiration was almost twice that seen in extrasynaptic mitochondria. Aging appears to affect the rate of ATP production in synaptic mitochondria differently than in extrasynaptic mitochondria. A decline in state III respiration is indicative of a decline in the rate of ATP production. The synaptic fraction also has the highest levels of oxidative stress in normal aging (Gilmer et al., 2010). Previous research has shown that increased oxidant formation and decreased proton force are associated with ATP production, which impairs both normal 
cellular activities and the ability to adapt to stress (Xiong et al., 2005).

The RCR takes into consideration the rate that ATP is produced (state III), as well as the basal metabolic rate that is not associated with ATP production (state IV). In this regard, RCR is a measurement of how coupled the ETC is to ATP production. RCR values declined significantly following injury in both extrasynaptic and synaptic mitochondria. In addition to the injury effect, synaptic mitochondria also displayed a significant aging effect. RCR values dropped from 8.5 to 5.5 (35\% reduction) in young animals, and to 4.1 in aged rats (53\% reduction). The extrasynaptic mitochondria showed a decline in RCR values of $15 \%$ in young animals and $18.7 \%$ in the older rats. Synaptic mitochondria appear to be not only more susceptible to damage than extrasynaptic mitochondria, but become increasingly more sensitive to perturbation with age. Findings of other researchers support the idea that synaptic mitochondria not only have different respiration capabilities than extrasynaptic mitochondria, but are more fragile as well (Brown et al., 2006; Davey and Clark 1996; Davey et al., 1997, 1998; Erecinska et al., 1996; Naga et al., 2007).

In the present set of experiments there was a significant alteration in both state III (ADP) and state V (FCCP) respiration states compared to that observed in the uninjured hemisphere, with the synaptic fraction demonstrating the greatest magnitude of change. One surprising finding was the modest change in state $\mathrm{V}$ (FCCP) respiration compared to that observed in state III (ADP) respiration. Normally FCCP state $\mathrm{V}$ respiration is significantly greater than state III respiration. There are a couple of reasons for the observed level of change in the state $\mathrm{V}$ respiration. FCCP, a pure uncoupler that acts as a protonophore, allows the protons built up in the inner membrane space to freely pass back into the matrix at a rapid rate. The oxygen utilization used in this process is dependent upon the concentration of FCCP used. Because the mitochondrial preparation used in these studies included a discontinuous Ficoll gradient in the purification, the amount of FCCP used may not have been adequate to obtain the maximal response. Alternatively, the diminished state $\mathrm{V}$ response may signal a problem with the adenine nucleotide transporter, or may indicate a problem with complex V of the ETC. This issue is currently under further investigation.

Most tissue types produce distinct amounts of free radicals, and therefore accumulate and respond to oxidative damage differently. With age the production of oxidants increases, and there is a decrease in antioxidant protection, and/or the damage oxidants inflict begins to exceed the cell's ability to repair the resulting damage (Butterfield et al., 2001). When the production of oxidants exceeds antioxidant defenses, the condition is known as oxidative stress, and this has is known to play a substantial role in accelerated aging, and in the damage seen after TBI (Ansari et al., 2008a, 2008b). Accumulation of damage to various complexes, proteins, and membranes, may explain this increased vulnerability to perturbations, and is supported by age-related differences in cortical tissue sparing seen after TBI (Shao et al., 2006).

Age-related increases in ROS can damage any structure within close proximity (e.g., proteins, lipids, and DNA). Oxidative damage accumulates with age and alters mitochondrial membrane proteins that can accelerate the production of ROS in aged animals in the presence of $\mathrm{Ca}^{2+}$ (Brown et al., 2004). These detrimental factors combine to produce a feasible theory on what drives the process of aging. Previously we reported significant age-related elevations in oxidative damage in the cortical synaptic mitochondrial fraction, but not extrasynaptic fraction, of naïve F344 rats (Gilmer et al., 2010). In the present study the contralateral uninjured hemisphere responded like naïve animals, with only the synaptic fraction manifesting age-related increases. The injured hemisphere responded quite differently, with both mitochondrial fractions displaying significant increases in each of the markers of oxidative stress. The most significant increases occurred in the synaptic fraction, with the aged cohort manifesting greater elevations than young animals. However, because of the elevated resting baseline in the aged animals, the injury-induced changes were actually less than those observed in the younger cohort. For example, injuryinduced PC levels in the synaptic fraction in younger animals increased $75 \%$, while in the aged cohort they only increased $24 \%$. Similarly, levels of $4-\mathrm{HNE}$ as a measure of lipid peroxidation were elevated by $34 \%$ in young animals after injury, but only $18 \%$ in the aged cohort. 4 -HNE is one of the most prevalent and toxic products generated during lipid peroxidation that form during oxidative stress (Renes et al., 2000), and it exerts its cytotoxic effects primarily by modifying intracellular proteins (Uchida et al., 1993). Levels of 3-NT were not elevated compared to the contralateral side in the aged cohort. These results suggest a possible upper limit for increases in oxidative stress in aging after TBI. This condition of higher levels of protein nitration and protein oxidation in the synaptic fraction may underlie the greater susceptibility of synaptic mitochondria to damage following trauma.

\section{Acknowledgments}

This work was supported by National Institutes of Health grant AG21981 and training grant NIH-NIDA 1 T32 DA 022738-01.

\section{Author Disclosure Statement}

No conflicting financial interests exist.

\section{References}

Adams, J.H., Graham, D.I., Murray, L.S., and Scott, G. (1982). Diffuse axonal injury due to nonmissile head injury in humans: an analysis of 45 cases. Ann. Neurol. 12, 557563.

Ansari, M.A., Roberts, K.N., and Scheff, S.W. (2008a). A time course of contusion-induced oxidative stress and synaptic proteins in cortex in a rat model of TBI. J. Neurotrauma 25, $513-526$

Ansari, M.A., Roberts, K.N., and Scheff, S.W. (2008b). Oxidative stress and modification of synaptic proteins in hippocampus after traumatic brain injury. Free Radic. Biol. Med. 45, 443-452.

Atamna, H., and Frey, W.H., 2nd (2007). Mechanisms of mitochondrial dysfunction and energy deficiency in Alzheimer's disease. Mitochondrion 7, 297-310.

Beal, M.F. (2005). Mitochondria take center stage in aging and neurodegeneration. Ann. Neurol. 58, 495-505.

Brown, M.R., Geddes, J.W., and Sullivan, P.G. (2004). Brain region-specific, age-related, alterations in mitochondrial responses to elevated calcium. J. Bioenerg. Biomembr. 36, 401-406. 
Brown, M.R., Sullivan, P.G., and Geddes, J.W. (2006). Synaptic mitochondria are more susceptible to Ca2+ overload than nonsynaptic mitochondria. J. Biol. Chem. 281, 1165811668.

Buki, A., Okonkwo, D.O., and Povlishock, J.T. (1999). Postinjury cyclosporin A administration limits axonal damage and disconnection in traumatic brain injury. J. Neurotrauma 16 , 511-521.

Bustamante, J., Czerniczyniec, A., Cymeryng, C., and Lores-Arnaiz, S. (2008). Age related changes from youth to adulthood in rat brain cortex: nitric oxide synthase and mitochondrial respiratory function. Neurochem. Res. 33, 1216-1223.

Butterfield, D.A., Howard, B.J., and LaFontaine, M.A. (2001). Brain oxidative stress in animal models of accelerated aging and the age-related neurodegenerative disorders, Alzheimer's disease and Huntington's disease. Curr. Med. Chem. 8, 815-828.

Casey, P.A., McKenna, M.C., Fiskum, G., Saraswati, M., and Robertson, C.L. (2008). Early and sustained alterations in cerebral metabolism after traumatic brain injury in immature rats. J. Neurotrauma 25, 603-614.

Chesnut, R.M., Ghajar, J., Maas, A.I.R., Marion, D.W., Servadei, F., Teasdale, G., Unterberg, A., von Holst, H., and Walter, B.C. (2000). Early indicators of prognosis in severe traumatic brain injury. J. Neurotrauma 17, 573-581.

Chiu, Y.J., and Richardson, A. (1980). Effect of age on the function of mitochondria isolated from brain and heart tissue. Exp. Gerontol. 15, 511-517.

Cocco, T., Sgobbo, P., Clemente, M., Lopriore, B., Grattagliano, I., Di Paola, M., and Villani, G. (2005). Tissue-specific changes of mitochondrial functions in aged rats: effect of a long-term dietary treatment with N-acetylcysteine. Free Radic. Biol. Med. 38, 796-805.

Coronado, V.G., Thomas, K.E., Sattin, R.W., and Johnson, R.L. (2005). The CDC traumatic brain injury surveillance system: characteristics of persons aged 65 years and older hospitalized with a TBI. J. Head Trauma Rehab. 20, 215-228.

Cotman, C.W., and Scheff, S.W. (1979). Compensatory synapse growth in aged animals after neuronal death. Mech. Ageing Dev. 9, 103-117.

Davey, G.P., and Clark, J.B. (1996). Threshold effects and control of oxidative phosphorylation in nonsynaptic rat brain mitochondria. J. Neurochem. 66, 1617-1624.

Davey, G.P., Canevari, L., and Clark, J.B. (1997). Threshold effects in synaptosomal and nonsynaptic mitochondria from hippocampal CA1 and paramedian neocortex brain regions. J. Neurochem. 69, 2564-2570.

Davey, G.P., Peuchen, S., and Clark, J.B. (1998). Energy thresholds in brain mitochondria. Potential involvement in neurodegeneration. J. Biol. Chem. 273, 12753-12757.

Dellinger, A.M., and Stevens, J.A. (2006). The injury problem among older adults: mortality, morbidity and costs. J. Safety Res. 37, 519-522.

Dencher, N.A., Frenzel, M., Reifschneider, N.H., Sugawa, M., and Krause, F. (2007). Proteome alterations in rat mitochondria caused by aging. Ann. N.Y. Acad. Sci. 1100, 291-298.

Deshmukh, D.R., Owen, O.E., and Patel, M.S. (1980). Effect of aging on the metabolism of pyruvate and 3-hydroxybutyrate in nonsynaptic and synaptic mitochondria from rat brain. J. Neurochem. 34, 1219-1224.

Driver, A.S., Kodavanti, P.R., and Mundy, W.R. (2000). Agerelated changes in reactive oxygen species production in rat brain homogenates. Neurotoxicol. Teratol. 22, 175-181.
Erecinska, M., Nelson, D., and Silver, I.A. (1996). Metabolic and energetic properties of isolated nerve ending particles (synaptosomes). Biochim. Biophys. Acta 1277, 13-34.

Fan, P., Yamauchi, T., Noble, L.J., and Ferriero, D.M. (2003). Age-dependent differences in glutathione peroxidase activity after traumatic brain injury. J. Neurotrauma 20, 437-445.

Feng, J., Xie, H., Meany, D.L., Thompson, L.V., Arriaga, E.A., and Griffin, T.J. (2008). Quantitative proteomic profiling of muscle type-dependent and age-dependent protein carbonylation in rat skeletal muscle mitochondria. J. Gerontol. A Biol. Sci. Med. Sci. 63, 1137-1152.

Finsterer, J. (2008). Cognitive decline as a manifestation of mitochondrial disorders (mitochondrial dementia). J. Neurol. Sci. $272,20-33$.

Flaada, J.T., Leibson, C.L., Mandrekar, J.N., Diehl, N., Perkins, P.K., Brown, A.W., and Malec, J.F. (2007). Relative risk of mortality after traumatic brain injury: a population-based study of the role of age and injury severity. J. Neurotrauma 24, 435-445.

Fukui, H., and Moraes, C.T. (2008). The mitochondrial impairment, oxidative stress and neurodegeneration connection: reality or just an attractive hypothesis? Trends Neurosci. 31, 251-256.

Gilmer, L.K., Ansari, M.A., and Scheff, S.W. (2010). Age-related changes in mitochondrial respiration and oxidative damage in the cerebral cortex of the Fischer 344 rat. Mech. Ageing Dev. 131, 133-143.

Gilmer, L.K., Roberts, K.N., Sullivan, P.G., Miller, K., and Scheff, S. (2009). Early mitochondrial dysfunction following cortical contusion injury. J. Neurotrauma 26, 1271-1280.

Gong, Y., Xi, G.H., Keep, R.F., Hoff, J.T., and Hua, Y. (2005). Aging enhances intracerebral hemorrhage-induced brain injury in rats. Acta Neurochir. Suppl. 95, 425-427.

Gouspillou, G., Bourdel-Marchasson, I., Rouland, R., Calmettes, G., Franconi, J.M., Deschodt-Arsac, V., and Diolez, P. (2009). Alteration of mitochondrial oxidative phosphorylation in aged skeletal muscle involves modification of adenine nucleotide translocator. Biochim. Biophys. Acta 1797, 143-151.

Greve, M.W., and Zink, B.J. (2009). Pathophysiology of traumatic brain injury. Mt. Sinai J. Med. 76, 97-104.

Hall, E.D., Detloff, M.R., Johnson, K., and Kupina, N.C. (2004). Peroxynitrite-mediated protein nitration and lipid peroxidation in a mouse model of traumatic brain injury. J. Neurotrauma 21, 9-20.

Hamm, R.J., Jenkins, L.W., Lyeth, B.G., White-Gbadebo, D.M., and Hayes, R.L. (1991). The effect of age on outcome following traumatic brain injury in rats. J. Neurosurg. 75, 916-921.

Hoane, M.R., Lasley, L.A., and Akstulewicz, S.L. (2004). Middle age increases tissue vulnerability and impairs sensorimotor and cognitive recovery following traumatic brain injury in the rat. Behav. Brain Res. 153, 189-197.

Hoch, F.L. (1992). Cardiolipins and biomembrane function. Biochim. Biophys. Acta 1113, 71-133.

Hukkelhoven, C.W., Steyerberg, E.W., Rampen, A.J., Farace, E., Habbema, J.D., Marshall, L.F., Murray, G.D., and Maas, A.I. (2003). Patient age and outcome following severe traumatic brain injury: an analysis of 5600 patients. J. Neurosurg. 99, 666-673.

Jacoby, S.F., Ackerson, T.H., and Richmond, T.S. (2006). Outcome from serious injury in older adults. J. Nurs. Scholarsh. 38, 133-140.

Joseph, J.A., Villalobos-Molina, R., Denisova, N., Erat, S., Cutler, R., and Strain, J. (1996). Age differences in sensitivity to H2O2or $\mathrm{NO}$-induced reductions in $\mathrm{K}(+)$-evoked dopamine release 
from superfused striatal slices: reversals by PBN or Trolox. Free Radic. Biol. Med. 20, 821-830.

Kaufmann, J.A., Bickford, P.C., and Taglialatela, G. (2001). Oxidative-stress-dependent up-regulation of Bcl-2 expression in the central nervous system of aged Fisher-344 rats. J. Neurochem. 76, 1099-1108.

Kovacs, E.J. (2005). Aging, traumatic injury, and estrogen treatment. Exp. Gerontol. 40, 549-555.

LaFrance, R., Brustovetsky, N., Sherburne, C., Delong, D., and Dubinsky, J.M. (2005). Age-related changes in regional brain mitochondria from Fischer 344 rats. Aging Cell 4, 139-145.

Langlois, J.A., Rutland-Brown, W., and Wald, M.M. (2006). The epidemiology and impact of traumatic brain injury: a brief overview. J. Head Trauma Rehab. 21, 375-378.

Leslie, S.W., Chandler, L.J., Barr, E.M., and Farrar, R.P. (1985). Reduced calcium uptake by rat brain mitochondria and synaptosomes in response to aging. Brain Res. 329, 177-183.

Lesnefsky, E.J., and Hoppel, C.L. (2006). Oxidative phosphorylation and aging. Ageing Res. Rev. 5, 402-433.

Lifshitz, J., Sullivan, P.G., Hovda, D.A., Wieloch, T., and McIntosh, T.K. (2004). Mitochondrial damage and dysfunction in traumatic brain injury. Mitochondrion 4, 705-713.

Long, J., Wang, X., Gao, H., Liu, Z., Liu, C., Miao, M., and Liu, J. (2006). Malonaldehyde acts as a mitochondrial toxin: Inhibitory effects on respiratory function and enzyme activities in isolated rat liver mitochondria. Life Sci. 79, 1466-1472.

Marklund, N., Salci, K., Ronquist, G., and Hillered, L. (2006). Energy metabolic changes in the early post-injury period following traumatic brain injury in rats. Neurochem. Res. 31, 1085-1093.

Maughan, P.H., Scholten, K.J., and Schmidt, R.H. (2000). Recovery of water maze performance in aged versus young rats after brain injury with the impact acceleration model. J. Neurotrauma 17, 1141-1153.

Meng, Q., Wong, Y.T., Chen, J., and Ruan, R. (2007). Age-related changes in mitochondrial function and antioxidative enzyme activity in Fischer 344 rats. Mech. Ageing Dev. 128, 286-292.

Merry, B.J. (2004). Oxidative stress and mitochondrial function with aging-the effects of calorie restriction. Aging Cell 3, 7-12.

Mosenthal, A.C., Lavery, R.F., Addis, M., Kaul, S., Ross, S., Marburger, R., Deitch, E.A., and Livingston, D.H. (2002). Isolated traumatic brain injury: age is an independent predictor of mortality and early outcome. J. Trauma 52, 907-911.

Mosenthal, A.C., Livingston, D.H., Lavery, R.F., Knudson, M.M., Lee, S., Morabito, D., Manley, G.T., Nathens, A., Jurkovich, G., Hoyt, D.B., and Coimbra, R. (2004). The effect of age on functional outcome in mild traumatic brain injury: 6-month report of a prospective multicenter trial. J. Trauma 56, 10421048.

Naga, K.K., Sullivan, P.G., and Geddes, J.W. (2007). High cyclophilin D content of synaptic mitochondria results in increased vulnerability to permeability transition. J. Neurosci. 27, 7469-7475.

Navarro, A., and Boveris, A. (2007). The mitochondrial energy transduction system and the aging process. Am. J. Physiol. Cell. Physiol. 292, C670-C686.

Navarro, A., Gomez, C., Sanchez-Pino, M.J., Gonzalez, H., Bandez, M.J., Boveris, A.D., and Boveris, A. (2005). Vitamin E at high doses improves survival, neurological performance, and brain mitochondrial function in aging male mice. Am. J. Physiol. Regul. Integr. Comp. Physiol. 289, R1392-R1399.

Navarro, A., Lopez-Cepero, J.M., Bandez, M.J., Sanchez-Pino, M.J., Gomez, C., Cadenas, E., and Boveris, A. (2008). Hippo- campal mitochondrial dysfunction in rat aging. Am. J. Physiol. Regul. Integr. Comp. Physiol. 294, R501-R509.

Nicholls, D.G. (2004). Mitochondrial membrane potential and aging. Aging Cell 3, 35-40.

Petrosillo, G., Fattoretti, P., Matera, M., Ruggiero, F.M., BertoniFreddari, C., and Paradies, G. (2008a). Melatonin prevents age-related mitochondrial dysfunction in rat brain via cardiolipin protection. Rejuvenation Res. 11, 935-943.

Petrosillo, G., Matera, M., Casanova, G., Ruggiero, F.M., and Paradies, G. (2008b). Mitochondrial dysfunction in rat brain with aging: Involvement of complex I, reactive oxygen species and cardiolipin. Neurochem. Int. 53, 126-131.

Povlishock, J.T., and Christman, C.W. (1995). The pathobiology of traumatically induced axonal injury in animals and humans: a review of current thoughts. J. Neurotrauma 12, 555-564.

Povlishock, J.T., Buki, A., Koiziumi, H., Stone, J., and Okonkwo, D.O. (1999). Initiating mechanisms involved in the pathobiology of traumatically induced axonal injury and interventions targeted at blunting their progression. Acta Neurochir. Suppl. 73, 15-20.

Renes, J., de Vries, E.E., Hooiveld, G.J., Krikken, I., Jansen, P.L., and Muller, M. (2000). Multidrug resistance protein MRP1 protects against the toxicity of the major lipid peroxidation product 4-hydroxynonenal. Biochem. J. 350 (Pt. 2), 555-561.

Richmond, T.S., Kauder, D., Strumpf, N., and Meredith, T. (2002). Characteristics and outcomes of serious traumatic injury in older adults. J. Am. Geriatr. Soc. 50, 215-222.

Richter, C. (1995). Oxidative damage to mitochondrial DNA and its relationship to ageing. Int. J. Biochem. Cell. Biol. 27, 647-653.

Robertson, C.L., Saraswati, M., and Fiskum, G. (2007). Mitochondrial dysfunction early after traumatic brain injury in immature rats. J. Neurochem. 101, 1248-1257.

Robertson, C.L., Scafidi, S., McKenna, M.C., and Fiskum, G. (2009). Mitochondrial mechanisms of cell death and neuroprotection in pediatric ischemic and traumatic brain injury. Exp. Neurol. 218, 371-380.

Rothweiler, B., Temkin, N.R., and Dikmen, S.S. (1998). Aging effect on psychosocial outcome in traumatic brain injury. Arch. Phys. Med. Rehabil. 79, 881-887.

Sastre, J., Pallardo, F.V., and Vina, J. (2003). The role of mitochondrial oxidative stress in aging. Free Radic. Biol. Med. 35, 1-8.

Scafidi, S., O'Brien, J., Hopkins, I., Robertson, C., Fiskum, G., and McKenna, M. (2009). Delayed cerebral oxidative glucose metabolism after traumatic brain injury in young rats. J. Neurochem. 109(Suppl. 1), 189-197.

Shah, S.A., Prough, D.S., Garcia, J.M., DeWitt, D.S., and Hellmich, H.L. (2006). Molecular correlates of age-specific responses to traumatic brain injury in mice. Exp. Gerontol. 41, 1201-1205.

Shao, C., Roberts, K.N., Markesbery, W.R., Scheff, S.W., and Lovell, M.A. (2006). Oxidative stress in head trauma in aging. Free Radic. Biol. Med. 41, 77-85.

Siesjo, B.K., and Siesjo, P. (1996). Mechanisms of secondary brain injury. Eur. J. Anaesthesiol. 13, 247-268.

Signorini, D.F., Andrews, P.J., Jones, P.A., Wardlaw, J.M., and Miller, J.D. (1999). Predicting survival using simple clinical variables: a case study in traumatic brain injury. J. Neurol. Neurosurg. Psychiatry 66, 20-25.

Stowe, D.F. (2009). Mitochondrial reactive oxygen species production in excitable cells: Modulators of mitochondrial and cell function. Antioxid. Redox. Signal. 11, 1373-1414. 
Sullivan, P.G., Keller, J.N., Bussen, W.L., and Scheff, S.W. (2002). Cytochrome $\mathrm{c}$ release and caspase activation after traumatic brain injury. Brain Res. 949, 88-96.

Sullivan, P.G., Keller, J.N., Mattson, M.P., and Scheff, S.W. (1998). Traumatic brain injury alters synaptic homeostasis: implications for impaired mitochondrial and transport function. J. Neurotrauma 15, 789-798.

Swerdlow, R.H. (2007). Treating neurodegeneration by modifying mitochondria: potential solutions to a "complex" problem. Antioxid. Redox. Signal. 9, 1591-1603.

Thompson, H.J., and Bourbonniere, M. (2006). Traumatic injury in the older adult from head to toe. Crit. Care Nurs. Clin. North Am. 18, 419-431.

Thompson, H.J., McCormick, W.C., and Kagan, S.H. (2006). Traumatic brain injury in older adults: epidemiology, outcomes, and future implications. J. Am. Geriatr. Soc. 54, 1590 1595.

Thurman, D.J., Alverson, C., Dunn, K.A., Guerrero, J., and Sniezek, J.E. (1999). Traumatic brain injury in the United States: A public health perspective. J. Head Trauma Rehabil. 14, 602-615.

Toescu, E.C., and Verkhratsky, A. (2003). Neuronal ageing from an intraneuronal perspective: roles of endoplasmic reticulum and mitochondria. Cell Calcium 34, 311-323.

Topp, K.S., Faddis, B.T., and Vijayan, V.K. (1989). Traumainduced proliferation of astrocytes in the brains of young and aged rats. Glia 2, 201-211.

Trifunovic, A., and Larsson, N.G. (2008). Mitochondrial dysfunction as a cause of ageing. J. Intern. Med. 263, 167-178.

Uchida, K., Szweda, L.I., Chae, H.Z., and Stadtman, E.R. (1993). Immunochemical detection of 4-hydroxynonenal protein adducts in oxidized hepatocytes. Proc. Natl. Acad. Sci. U.S.A. 90, 8742-8746.

Utomo, W.K., Gabbe, B.J., Simpson, P.M., and Cameron, P.A. (2009). Predictors of in-hospital mortality and 6-month functional outcomes in older adults after moderate to severe traumatic brain injury. Injury 40, 973-977.
Van Remmen, H., and Richardson, A. (2001). Oxidative damage to mitochondria and aging. Exp. Gerontol. 36, 957-968.

Victorino, G.P., Chong, T.J., and Pal, J.D. (2003). Trauma in the elderly patient. Arch. Surg. 138, 1093-1098.

Vollmer, D., Torner, J.C., Jane, J.A., Sadovnic, B., Charlebois, D., Eisenberg, H.M., Foulkes, M.A., Marmarou, A., and Marshall, L.F. (1991). Age and outcome following traumatic coma: why do older patients fare worse? J. Neurosurg. 75, S37-S49.

Walker, L.M., York, J.L., Imam, S.Z., Ali, S.F., Muldrew, K.L., and Mayeux, P.R. (2001). Oxidative stress and reactive nitrogen species generation during renal ischemia. Toxicol. Sci. 63, 143-148.

Werner, C., and Engelhard, K. (2007). Pathophysiology of traumatic brain injury. Br. J. Anaesth. 99, 4-9.

Xiong, Y., Gu, Q., Peterson, P.L., Muizelaar, J.P., and Lee, C.P. (1997). Mitochondrial dysfunction and calcium perturbation induced by traumatic brain injury. J. Neurotrauma 14, 23-34.

Xiong, Y., Shie, F.S., Zhang, J., Lee, C.P., and Ho, Y.S. (2005). Prevention of mitochondrial dysfunction in post-traumatic mouse brain by superoxide dismutase. J. Neurochem. 95, 732-744.

Yager, J.Y., Wright, S., Armstrong, E.A., Jahraus, C.M., and Saucier, D.M. (2006). The influence of aging on recovery following ischemic brain damage. Behav. Brain Res. 173, 171180.

Zhu, W., Umegaki, H., Shinkai, T., Kurotani, S., Suzuki, Y., Endo, H., and Iguchi, A. (2003). Different glial reactions to hippocampal stab wounds in young adult and aged rats. J. Gerontol. A Biol. Sci. Med. Sci. 58, 117-122.

Address correspondence to: Stephen W. Scheff, Ph.D.

Sanders-Brown Center on Aging University of Kentucky

101 Sanders-Brown Building Lexington, KY 40536-0230

E-mail: sscheff@email.uky.edu 